\title{
Actinomycosis of the parotid gland. Challenges in diagnosis and treatment - case report
}

\author{
C. Ivanova, S. Bachurska, K. Popov \\ University specialized hospital for active treatment in oncology, Sofia - Department of Pathology
}

\begin{abstract}
Introduction: Primary actinomycosis of the parotid gland is a rare infectious disease which can mimic a malignant process both clinically and diagnostically.

Case report: We present a case report of actinomycosis of the parotid gland manifesting as a slowly growing tumor in the left neck region. Methods: Computer tomography, histopathologic examination

Results: Total sialoadenectomy. Confirmed diagnosis of actinomycosis. Patient introduced to an infectious disease specialist.

Conclusion: Although this location of actinomycosis is rare, the disease must be considered in the differential diagnosis of parotid gland tumors.

Key words: actinomycosis, parotid gland
\end{abstract}

\section{Introduction}

Actinomycosis is a bacterial infection caused by Gram positive, branching filamentous bacilli of Actinomyces family. The course of the disease is subacute or chronic suppurative - the characteristic granulomatous inflammation initially forms abscesses which later result in fistulae or sinus tracts involving adjacent tissues. (1) The most common causative agent is Actinomyces israelii, a saprophyte usually colonazing oral cavity, gastrointestinal tract and vagina in humans. (2) Under normal conditions the susceptibility to actinomycosis is low but exogenous or endogenous factors compromising host' $\mathrm{s}$ anatomical barriers and local immunity (such as surgical interventions, trauma or foreign bodies) could induce pathogenic activity of the microorganism. (3)

Actinomycosis occurs with higher prevalence rates in geographic areas of low socioeconomic status. For unkown reason the disease is more common in men as male to female ratio is 3:1. Age range includes patients from 20 to 50 years old - immunocompetent and immunocompromised.

Three basic forms of the disease are classified as follows - cervicofacial (50-70\%), thoracic (15$20 \%$ ) and abdominopelvic (10-15\%). (1)

Cervicofacial actinomycosis, the most common clinical form, is Well described in the literature. (1) However, the reported cases concerning its location 
in the parotid gland remain few.

\section{Case report}

A 65- year old male patient was admitted to Otorhinolaryngology department of University specialized hospital for active treatment in oncology, Sofia for the first time in May 2020. He presented with gradually progressing swelling in left neck region dating from January 2020. Physical examination showed formation with maximum diameter 5-6 $\mathrm{cm}$, well circumscribed and palpatory mobile. The patient did not report any painful or tingling sensations related to the tumor.

\section{Methods}

CT scan revealed a solid calcified tumor located in lower third of the left parotid gland which enhanced its densitometric features after administration of contrast. Right parotid gland, submandibular glands bilaterally, pharynx and larynx - reserved anatomic structure. Enlarged lymph nodes were not identified. Clinical suspicion of malignancy imposed the performance of total sialoadenectomy. Operative material consisted of a tumor $5 \times 2 \mathrm{~cm}$ affecting parotid gland tail and deep part. After lymph node dissection in second and third neck segment the main resectate was sent for histopathologic examination.

Microscopic slides revealed abundant inflammatory infiltrate composed of lymphocytes, neutrophils, giant cells and fibroblasts resulting in the formation of a granuloma. (fig. 1) The specific bacterial colonies are seen as intensely basophilic clumps with irradiating filamentous eosinophilic borders when stained with hematoxylin - eosin (HE). (fig. 2) Examined lymph nodes showed normal histologic structure.

\section{Results}

In the presented case report the patient had neither identified risk factors, nor an immunocompromised state. After successful surgical removal of the tumor a consultation with infectious disease specialist was performed.

\section{Discussion}

The earliest cases of parotid gland actinomycosis were reported by Leonarmant in 1913; Beck in 1922 and Laca - 1961. A few years later - in 1973 Hopkins conducted a detailed review of his scientific results in relation to the disease. (5) Considered a rare infection nowadays, actinomycosis should not be excluded from the differential diagnosis of parotid glands tumors. (6)

Actinomyces israelii is a commensial microorganism inhabiting tonsillar crypts, dental caries and plaques, periodontal space. $(1,6)$ Predisposing factors for cervicofacial actinomycosis are processes causing disruption of anatomical barriers such as traumas, surgical interventions or dental procedures. (5) Due to the relatively low virulence of Actinomyces israelii co-pathogenic bacterial flora producing toxins and enzymes is required in order to compromise local immune response. Aggregatibacter actinomycetemcomitans, Peptostreptococcus, Prevotella, Fusobacterium, Bacteroides, Staphylococcus, Streptococcus, Enterobacteriaceae are the most common co-pathogens. $(7,10)$ Actinomycosis is considered mainly an endogenous infection - possible route of bacterial spread could be in retrograde direction trough Stensens's duct (primary actinomycosis); from adjacent tissues (secondary actinomycosis) or in rare cases trough hematogenous dissemination. (5)

Clinical presentation of the disease does not differ significantly from that in other locations of cervicofacial actinomycosis. (8) It debutes as well defined, firm mass (abcess) usually involving the submandibular or perimandibular region; over time fluctuation appears and multiple sinus tracts are formed draining exudate with "sulphur granules" - bacterial colonies. Overlying skin could be erythematous. Pain syndrome, facial nerve involvement and neck lymphadenopathy are not usual. (7) Imaging studies such as computer tomography and echography reveal the presence of poorly defined masslike lesion invading surrounding tissues features raising suspicion of possible malignancy. $(5,6)$ Definitive diagnosis could be made after identification of the characteristic bacterial colonies (sulphur granules) in cytological, histological samples or after microbiological examination. (9) Differential diagnosis of painless parotid tumor like lesions should include other nosological entities presented in table 1 . 
Tabl. 1. Differential diagnosis of painless tumor like lesions of the parotid gland

\begin{tabular}{|l|}
\hline 1. Neoplasms: \\
- Benign- pleomorphic adenoma, Warthin tumor, \\
facial nerve schannoma \\
- Malignant- mucoepidermoid carcinoma, adenoid- \\
cystic carcinoma, lymphoma \\
\hline 2. Chronic inflammation: \\
- Mixed infections \\
- Actinomycosis \\
\hline 3. Granulomatous inflammation: \\
- Tuberculosis \\
- Leprosy \\
- Syphilis \\
- Felinosis \\
4. Mycotic infections: \\
- Histoplasmosis \\
- Coccidiomycosis \\
- Cryptococcosis \\
- Mucormycosis \\
\hline 5. Osteomyelitis. \\
6. Branchial cysts. \\
7. Parapharyngeal tumor. \\
8. Sjogren's syndrome.
\end{tabular}

Treatment consists of antimicrobial therapy with drug of choice Penicillin G or alternative - macrolide. (7) In some cases clinically important co-infecting bacteria are targeted with another specific antibiotic. (10) Standard duration of the therapy is 6 to 12 months but variations are possible when considering individual clinical course. Surgery can be adjunctive in selected cases - those with formed abscesses and fistulas. (11) Possible complications of the untreated infection are osteomyelitis, brain abscesses, chronic meningitis, hepatic actinomycosis and polyorgan deficiency due to dissemination.

\section{Conclusion}

Although considered a relatively rare infection, actinomycosis should not be neglected as diagnosis. Differentiation from a true malignant process is crucial for adequate treatment. Prognosis of the disease is positive and prevention consists of maintaining proper oral hygiene.

\section{References:}

1. Dubey A, Murthy J, Banerjee P. Actinomycosis of the parotid gland. Indian J Otolaryngol Head Neck Surg. v. 56 (4); 2004 p. $306-308$.

2. Ferry T, Valour F, Karsenty J, Breton P, Gleizal A, Braun E. Actinomycosis: etiology, clinical features, diagnosis, treatment, and management. Infection and Drug Resistance. 2014; (7): 183 - 197.

3. Ritter F. A clinical study of actinomycosis. Arch Otolaryngol. 1961; 74 (3): 314-322.

4. Sharma S, Hashmi M, Valentino D. Actinomycosis [Internet]. Ncbi. nlm. nih. gov. 2020 [cited 2020 Jan 4]. Available from: https://www.ncbi. nlm.nih.gov/books/NBK482151/

5. Ulvi B. Actinomycosis of the parotid gland. Plastic and Reconstructive Surgery. 2005; 115 (2): 659-661.

6. Sittitrai P, Srivanitchapoom C, Pattarasakulchai T, Lekawanavijit S. Actinomycosis presenting as a parotid tumor. Elsevier Ireland Ltd.; 2011 ; 39 (2): 241-3.

7. Okulicz J, Polenakovik H, Polenakovik S. Actinomycosis: Background, Pathophysiology, Epidemiology [Internet]. Emedicine. medscape. com. 2020 [cited $2020 \mathrm{Jul} 5$ ]. Available from: https://emedicine.medscape.com/article/211587-overview

8. Sanzama L. Actinomycosis of the parotid gland: report of five cases. Elsevier Inc.; 1965;19 (2); 197-204.

9. Varghese B, Sebastian P, Ramachandran K, Pandey M. Actinomycosis of the Parotid Masquerading as Malignant Neoplasm. BMC Cancer; 2004; 4:7.

10. Boyanova L, Kolarov R, Mateva L, Markovska R, Mitov I. Actinomycosis: a frequently forgotten disease. Future Microbiology. 2015; 10(4): 613-628.

11. Boyanova L, Sabov R, Kolarov R, Mitov I. Chronic odontogenic osteomyelitis and facial actinomycosis of six-month duration. JMM Case reports; 2014.

The present case report has not been published or submitted for publication elsewhere. 